\title{
Digital Technology for Assessing the Color of Textile Materials
}

\author{
Gerasimenko Nicolay I., Raykova Elena Y
}

\begin{abstract}
One way to express yourself in clothes is through color. Color is a tool of influence. It determines the aesthetic properties of clothing. At the same time, the nature of color is physical, which allows for an objective assessment. The proposed article substantiates the possibility of using a flatbed scanner to measure the color characteristics of textile materials. This topic is very relevant today, which makes the work useful for professionals interested in studying the characteristics and color stability of textile materials. The presented method differs in the speed of data processing. All information about the color characteristics of product samples can be obtained in one test. Experimental data are presented in digital form, which increases the accuracy and reduces the complexity of further processing.
\end{abstract}

Keywords: BMP files, color, materials, scanner, textiles.

\section{INTRODUCTION}

C olor is the property of optical radiation to induce a certain visual action in accordance with the spectral composition of this radiation. The color of natural bodies is determined by the spectral composition of optical radiation emanating from them. The predominance of certain wavelengths in the spectrum creates a sense of "color". This is because the effects of different wavelengths on the eye are not the same. For example, the sensation of "white" color occurs when the radiation wavelengths are distributed more or less evenly. This is what we see in daylight.

Color belongs exclusively to the field of perception. It is determined by human feelings. Color assessment is determined by both the properties of the perceived light and the visual perception system. It is this system that creates the perception of color. This in turn introduces an element of subjectivity to the definition of color. The visual perception system includes: the eyes, nerves, and visual areas of the brain that process and analyze signals from the eyes. It can be characterized by spectral sensitivity. Spectral sensitivity is the dependence of the magnitude of the signal arising in the eye receptors on the wavelength of the perceived radiation. Color vision is due to the presence of light-sensitive receptors in the eye with selective spectral sensitivity. These are the so-called

Revised Manuscript Received on December 20, 2020.

*Correspondence Author

Gerasimenko Nicolay I*, department of Physics, Plekhanov Russian University of Economics, Moscow, Russia. Email n_i_ger @ mail.ru.

Raykova Elena Y., department of Commodity Science, Plekhanov Russian University of Economics, Moscow, Russia. Email: Raykova.EY@rea.ru.

(c) The Authors. Published by Blue Eyes Intelligence Engineering and Sciences Publication (BEIESP). This is an open access article under the CC BY-NC-ND license (http://creativecommons.org/licenses/by-nc-nd/4.0/) cones [1]. There are cones with high spectral sensitivity in the yellow-orange, blue and green regions of the visible range. In addition, the eye contains sensory receptors with a wide spectral range of sensitivity (rods). Their signals are interpreted by the brain as grayscale. All other things being equal, different observers may evaluate the same color differently. This is determined by the relative number of different types of cones present in the eye. Congenital disease - color blindness is caused by the absence (or an abnormally small number) of receptors sensitized to any of the primary colors. The second significant factor affecting the determination of color is the spectral composition of the light rays illuminating the object under study. Let's assume we are using white light. Then each spectral harmonic of the light signal will interact differently with the object under study. As a result, a wave appears with a spectrum that is most likely different from the initial one. This will be perceived by the visual system as a certain color. It is because of the difference in the spectral composition of the lighting that the same pattern appears to be different depending on whether it is illuminated by daylight or an incandescent lamp. Therefore, to obtain the most accurate readings, a source with a standard spectral content and standard brightness should be used for color measurements. [2] The color is completely and unambiguously determined by the spectrum obtained after the interaction of the standard light beam with the object of interest. Human vision is a three-color analyzer plus an intensity analyzer. For such an analyzer, light rays of different spectral composition can exist, giving the perception of one color. This phenomenon is called metamerism. Colors that have a different spectral composition, but give the same visual perception, are called metameric. In printing, the presence of metamerism leads to the fact that in some lighting conditions, colors may appear the same, and in others - different. It is of interest to develop objective methods for determining the color characteristics of textile materials. At the same time, the most promising seems to be the use of information technologies for their assessment [3].

\section{LITERATURE REVIEW}

Mixing light rays of different colors results in a new color. This phenomenon was investigated by Isaac Newton [4]. Based on his research, Newton formulated four guidelines for color mixing:

1. An additional color is uniquely identified for each color. When mixed with an additional color, an achromatic gray is obtained.

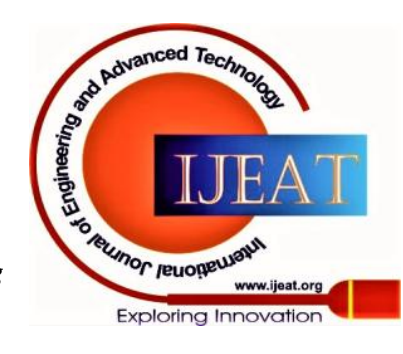




\section{Digital Technology for Assessing the Color of Textile Materials}

2. Subjectively equally perceived colors when mixed with other colors give equally perceived colors, regardless of their spectral composition.

3. When two different colors are mixed, the resulting color is intermediate between the original colors.

By mixing this intermediate color with one of the original, it is impossible to obtain a second original color.

4. When you mix two different colors, the resulting color will always be less saturated than at least one of the original colors.

Based on these laws, in the mid-19th century, Hermann Grassmann (1809 -1877) created the International Color Specification System in terms of the Standard Observer (CSS-31). Grassmann's general ideas about abstract vector spaces led him to the discovery of an important fact - the ability to consider color sensations as three-dimensional vectors. This is now the foundation of modern color theory. He also established (1853) the laws of color addition. Grassmann based his system on four main principles, known in the domestic literature as Grassmann's laws:

1. Color is three-dimensional. Any color can be obtained by mixing no more than three primary colors (they are also called color coordinates);

2. A continuous change in the constituent colors leads to a continuous change in the resulting color;

3. Two identical colors (even if they are metameric) when mixed with a third give the same color;

4. Colors are additive. This means that the coordinates of the blended color are equal to the sum of the coordinates of the blended colors. [5]

The choice of primary colors is largely arbitrary. Any system based on three primary colors correctly identifies any color. The system for defining a color with color coordinates allows you to form the concept of a color space.

The most natural from the point of view of physiology is the system in which blue, green and red are used as the main colors. This system is called RGB (from the English. Red, Green and Blue). RGB space is used in the design of various display systems. In this space, the addition of equal amounts of primary colors always gives an achromatic color, perceived by the eye as white or a shade of gray. RGB is a light combining system. Colors are added on a black background. An example of this addition would be colors obtained from theater spotlights.

When printed, the image is usually built on a white background. A dye applied to white paper (or fabric) absorbs individual harmonics of the incident white light. The result is colored reflected light rays. In this sense, it is said that in printing, the formation of an image occurs by subtracting colors. The most used color in printing is black. In RGB space, black arises from the complete absence of color components.

Obviously, it is inconvenient to use the RGB system in printing. The printing industry uses the CMY system. It uses cyan, magenta, and yellow. They complement red, green and blue. Adding equal proportions of primary colors in this system also results in shades of gray. However, unlike the system, an increase in the coordinates of the primary colors leads not to lightening, but to a darkening of the gray, which, with sufficient intensity of the primary colors, becomes almost black. The absence of dyes in this system is equivalent to the white color of an unpainted background.

The CMY system is used, in particular, in color printers. The downside of this system is the high consumption of expensive dyes required to form black. In addition, the slightest change in the proportions of the dyes results in unwanted shades of gray. (This phenomenon is similar to RGB white balance.) Therefore, the CMY system is almost completely replaced by the CMYK (cyan, magenta, yellow and black) system, The CMYK system differs from CMY by the presence of the fourth color — black. This makes it possible to achieve a significant reduction in the cost of printing materials by saving basic dyes.

The described color measurement systems are natural and have a simple theoretical interpretation. However, in practical applications such as visual color selection, these systems are inconvenient, primarily because their results do not match the emotional assessment of the color. When choosing a color, it is more convenient to follow the scheme used in acoustics.

In acoustics, sound is characterized by three dimensions: pitch, timbre, and volume. Pitch refers to the frequency of the fundamental spectral harmonic, that is, the harmonic that has the highest energy. The notorious ear for music is the ability to highlight this main harmonic in a complex sound wave. Timbre characterizes the distribution of energy over the harmonics of the wave, that is, its spectrum. For example, the sounds of a European's speech have the same tone, but differ in spectrum. Loudness characterizes the integral energy transmitted by the wave to the receiver per unit of time.

The HSV system used in color selection is similar to that used in acoustics. Color in this system is characterized by hue, saturation, and amount of light (hue, saturation, value). Currently, the main color measurement system is the MKO system, which uses abstract color coordinates. They differ from RGB coordinates by the presence of negative values that do not correspond to any real color composition.

\section{RESEARCH METHODS}

The main target of our investigation is to create an original method for determining the distributed color characteristics of textile materials using information technologies for their assessment. Currently, there are many modern physical and physicochemical methods for determining color. All methods according to their capabilities can be conditionally divided into two types:

1. Allowing analyzing and studying subtle chemical and physical changes occurring with polymer fiber and when the fiber interacts with dyes,

2. Allowing evaluating only color characteristics in textiles.

Both types include a number of techniques such as absorption and fluorescence spectroscopy, polarized light microscopy, molecular weight distribution, thermal analysis.

In order to describe the color characteristics of various fabrics, we wanted to be able to characterize color not only subjectively but also objectively.

Published By:

Blue Eyes Intelligence Engineering and Sciences Publication

(C) Copyriaht: All riahts reserved.

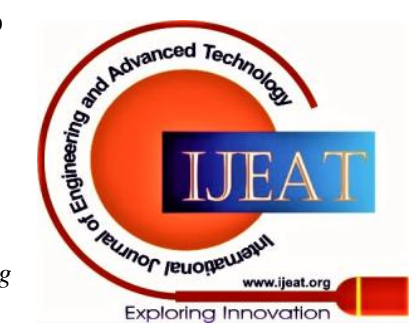


At present, spectrophotometers, color comparators and colorimeters are mainly used to objectively study the characteristics of textile materials. To determine the color characteristics of fabrics, the national standard $\mathrm{R}$ ISO 105-A03-99 [10] recommends using a spectrophotometer or a standard colorimeter.

The use of a spectrophotometer (a very expensive device) is certainly not justified as it lacks a clear correspondence between color and spectrum. This ambiguity is caused by the phenomenon of metamerism (the existence of identical colors with different spectral composition).

Modern colorimeters designed to measure tissue color use the measurement of light from a reference light source scattered by the sample under study [11]. These devices also have several disadvantages. First of all, such devices should be equipped with a separate reference light source. They require preliminary calibration ("white balance") and have a relatively small aperture of the input light beams. The limited aperture results in color being detected locally over a small area of the sample. Therefore, a large number of laborious measurements over the entire surface of the sample are required to determine the unevenness of its color and further statistical processing of the results. At the same time, I would like to easily get the average color characteristic of the entire sample.

\section{RESULTS AND DISCUSSION}

A judgment on the color properties of a textile material can be made by analyzing its digital image. (In case it is made with a precise light sensor and a calibrated white light source). The thickness of the textile material is many times smaller than its other dimensions. Therefore, it is convenient to use a flatbed scanner to measure the color characteristics of textile materials. It contains a standard light source with the required color temperature and a line of receiver sensors that are sensitive to different color coordinates in accordance with the requirements of the standard. The result of the scanner operation is contained in a file of one of the popular graphic formats, from where the color coordinates of each point of the image can be extracted with special software.

A scanner is a device that reads information from flat media and converts it into digital form suitable for processing on a computer. It is characterized by optical and interpolated resolution, maximum readable sample size, and recorded color depth.

Optical resolution is the size of the smallest part of the image that the scanner can recognize. (Usually it is a fraction of a millimeter). It's measured in dots per inch. Integral resolution is determined by optical and additional mathematical processing, which allows extrapolation to increase the "registered" number of registered points. In experimental work, such extrapolation should be avoided. The color depth determines the maximum value of the color coordinates and, therefore, the possible number of recorded colors.

Apart from technical details, we can note that regardless of the design features of each particular scanner, it is designed so that its color rendition meets the requirements of the standard.
This means that the scanner can serve as a technical base for registering the color of a textile material.

The color information of the object being scanned is encoded by the scanner driver in the output file. These files come in a variety of formats. Most often it is jpg, tiff or bmp format.

The jpg format was used in older scanners because it produces smaller files. Unfortunately, decreasing the file size in this case is accompanied by the loss of information. This format should not be used for scientific purposes. The tiff format is preferred. The disadvantage of this type of files is their relatively difficult encoding. In addition, there are several types of tiff files, differing in the details of the internal device. The easiest way to encode color information is in a .bmp file.

BMP is the original graphics format adopted in the MS Windows operating system. A file of this format consists of a header, an information block with a color bitmap, if any, and an information part containing the color coordinates of each of the pixels or a link to these coordinates. The header has a fixed length of 14 bytes and can be omitted when parsing color. However, in order to avoid mistakes, it is desirable to control some of its fields. This will avoid incorrect results from the damaged file.

At the beginning of the header is a two-byte field labeled BM. Its absence means that you have an unknown file format. The next field is four bytes. It is also very useful as it contains the length of the file in bytes. The next 4 bytes are reserved and must contain zeros. Finally, the last header field is critical because it contains the offset in the encoded data area. [6]

This work used an HP Scanjet 5590P A4 flatbed scanner. The light receptors of this scanner are equipped with calibrated light filters that provide color registration accuracy sufficient to solve not only printing, but also scientific problems. The result of the scan is information about the distribution of color on the sample $20 \times 30 \mathrm{~cm}$. The scanner provides a geometric resolution of up to $600 \mathrm{dpi}$. This makes it possible to draw conclusions about the color of individual small elements of the material and, therefore, it is easy to control the color distribution over the sample. Information about the distribution of color in the sample is issued in the form of a graphic file. Its format is determined by the scanner manufacturer.

Statistical processing is ensured by the high graphic resolution of the scanner. It allows you to extract additional color characteristics that are not available when measured with a standard color meter. The disadvantages of measuring color with a flatbed scanner include the difficulty of decoding the extracted information. For example, BMP files may contain a series of zeros at the end of a bitfield string. If they are not removed, this will lead to incorrect operation of the processing program.

The processing programs were compiled by us taking into account the specified features of the file. The choice of the programming language was determined by the systemic orientation of the work (analysis of graphic files),

Published By:

Blue Eyes Intelligence Engineering and Sciences Publication (C) Copyright: All rights reserved.

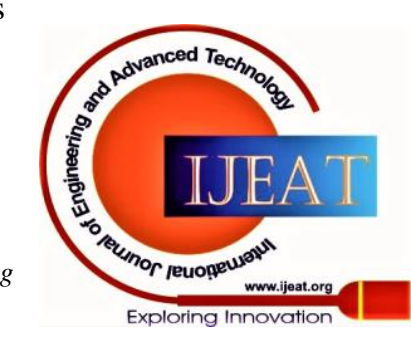




\section{Digital Technology for Assessing the Color of Textile Materials}

as well as the availability and efficiency of the programming environment. The focus of the study made it possible to make a choice in favor of the system-oriented $\mathrm{C}$ language in its simplest version (without using abstract data types and user-defined classes). Microsoft Visual Studio 2008 is used as a programming environment.

The choice is explained by both the professional level of the compiler and the availability of its licensed version.

To achieve the set goals, a simple express processing of a graphic file is used, which is not associated with any graphic constructions. Therefore, the console model of the program was chosen. It should be noted here that a Windows console application differs from a window application only in the way it presents information, while remaining event-oriented. [7]

The program has a modular structure and includes a root module, a file selection module, a header decryption module, a color information selection module (including creating a table for constructing a histogram of the distribution of each of the primary colors) and a presentation module. The results of the program's operation are displayed on the screen accompanied by text comments, and also displayed in parallel to a disk file in the directory containing the program module. The latter allows the use of standard programs from the Microsoft Office package for plotting graphs and further statistical processing. The output file has a txt extension.

Samples of cotton and knitted fabrics were taken for the study. The method of detecting chromaticity using a scanner requires a fairly flat surface of the material, since folds and creases in the fabric can distort the image due to the resulting glare. To avoid this, the samples should be thoroughly ironed before measurements $[8,9]$.

The measurement process itself is reduced to scanning the surface of the material under study. The scan result as a tiff or bmp file is further processed using a specialized program. Color coordinates that characterize the color of individual points of the test sample are output to a file in text format. Integral characteristics of the sample are also displayed there: average color coordinates and their variance. The text format of the output file makes it easy to import it into an office program for further statistical processing. First of all, for constructing distribution functions of color coordinates by value. A meaningful analysis of these functions makes it easy to draw conclusions about the influence of various factors (washing, solar radiation, etc.) on the color of the fabric

\section{CONCLUSIONS}

The work has shown that this digital technology is suitable for assessing the color of textile materials. Its main advantage is that it makes it possible to obtain a large amount of statistical data (several thousand). In addition, this technology enables fast data processing. All information about the sample can be obtained in one pass. The information is presented in digital form, which reduces the complexity of further processing. Information is processed on a large sample at a time. The prospects of the presented work depend on the improvement of this technology by improving the processing software and conducting market research aimed at determining the resistance of the fabric to fading, washing, etc.

\section{REFERENCES}

1. Gerasimenko N.I., Raykova E.Y., Gerasimenko T.N., "Modern methods of researching the quality of clothing (monograph) (book style with the title of the article and the editor)", LAP LAMBERT Academic Publishing, Germany, 2013, p. ... 15-20.

2. Raykova E.Y., Tsybanina I.V., Application of factor analysis in assessing the quality of clothing (Periodic style) - Moscow, Design and technology, 2018, No. 68 (10), p. 36-46.

3. Pekhtasheva E.L., Raykova E.Y. Improving approaches to the classification of clothing identification (Periodic style). - Moscow, Design and Technologies, 2020, No. 75 (117), p. 86-95.

4. Isaaci Newtoni, Lectiones opticale - EX Officina academiae scientiarvm FRSS MCMXLVI.

5. Johannes Itten The Art of Color. (Book style with article title and editor), Moscow, 2004, p. 12-27, 32-56.

6. Romanov V.Yu. Popular file formats for storing graphics on an IBM PC (book style with article title and editor). M .: Unitex, 1992.

7. Gerasimenko NI, Raykova E.Y., Gerasimenko TN, On the possibility of using information technology to study the color of complex systems [Dig. XXI Int. Conf. Electromagnetic field and materials, National Research University MPEI, Russia, 2013, p. 294].

8. Russian Standard GOST R ISO 105-A03-99 Textiles. Color fastness tests. Part A03. Gray scale for assessing staining. - Standardinform: Moscow, Russia, 2010.

9. On the safety of light industry products. Technical Regulations of the Customs Union (TR CU 017/2011). Decision of the Customs Union Commission dated December 9, 2011 No. 876.

\section{AUTHORS PROFILE}

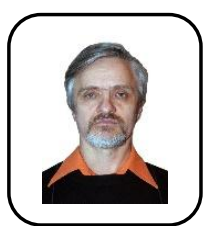

Gerasimenko Nikolai I. graduated from Moscow State University. M.V. Lomonosov as a physicist in 1970. In 1978 he completed his postgraduate studies at this university. In 1979 he received a PhD in Physics and Mathematics. Associate professor since 1980. He worked at the Plekhanov Russian University of Economics. Research interests - molecular physics and applied physical optics. In parallel, he taught a course in applied and system programming. Author of 120 publications, including 2 monographs, 2 textbooks.

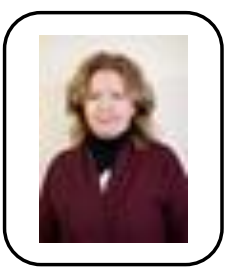

Raykova Elena Y. works as an associate professor of the Department of Commodity Science at the Plekhanov Russian University of Economics, Moscow, Russia. PhD (Technical Science). Thesis theme: "Influence of yarn properties upon cotton fabrics". Professional interests concern with commodity research, conformity assurance of products and services, consumer protection, product safety, commodity properties, quality management, technical regulation and standardization. Author of 168 publications, including 5 textbooks, 1 monograph.
Published By:

Blue Eyes Intelligence Engineering and Sciences Publication

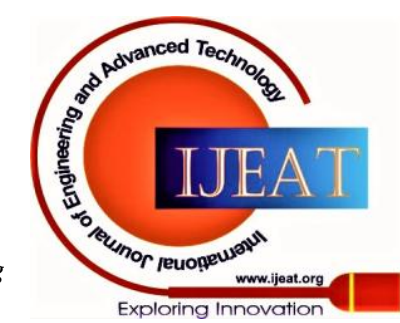

